\title{
Theory of nonlinear phononics for coherent light control of solids
}

\author{
Alaska Subedi, ${ }^{1}$ Andrea Cavalleri, ${ }^{2,3}$ and Antoine Georges ${ }^{1,4,5}$ \\ ${ }^{1}$ Centre de Physique Théorique, École Polytechnique, CNRS, 91128 Palaiseau Cedex, France \\ ${ }^{2}$ Max Planck Institute for the Structure and Dynamics of Matter, Hamburg, Germany \\ ${ }^{3}$ Department of Physics, Oxford University, Clarendon Laboratory, Parks Road, Oxford, United Kingdom \\ ${ }^{4}$ Collège de France, 11 place Marcelin Berthelot, 75005 Paris, France \\ ${ }^{5}$ DPMC-MaNEP, Université de Genève, CH-1211 Genève, Switzerland
}

(Received 3 November 2013; revised manuscript received 2 April 2014; published 11 June 2014)

\begin{abstract}
We present a microscopic theory for ultrafast control of solids with high-intensity terahertz frequency optical pulses. When resonant with selected infrared-active vibrations, these pulses transiently modify the crystal structure and lead to new collective electronic properties. The theory predicts the dynamical path taken by the crystal lattice using first-principles calculations of the energy surface and classical equations of motion, as well as symmetry considerations. Two classes of dynamics are identified. In the perturbative regime, displacements along the normal mode coordinate of symmetry-preserving Raman active modes can be achieved by cubic anharmonicities. This explains the light-induced insulator-to-metal transition reported experimentally in manganites. We predict a regime in which ultrafast instabilities that break crystal symmetry can be induced. This nonperturbative effect involves a quartic anharmonic coupling and occurs above a critical threshold, below which the nonlinear dynamics of the driven mode displays softening and dynamical stabilization.
\end{abstract}

DOI: 10.1103/PhysRevB.89.220301 PACS number(s): 78.47.J-, 63.20.Ry, 71.15.Mb, 71.30.+h

The use of light to control the structural and electronic properties of solids is emerging as an area of great interest for both basic research and potential applications. Much work has been dedicated to materials with strong electronic correlations, which exhibit remarkable collective properties such as superconductivity, ferroelectricity, or colossal magnetoresistance, and can be switched by illumination with light [1-5].

Recently, the possibilities of materials control by light have been greatly expanded by the demonstration of phase switching through selective vibrational excitation, that is, by deforming the lattice along a specific normal-mode coordinate [6-8]. Let us mention, in particular, the achievement of light-induced superconductivity [9-11]. Mode-selective optical control is especially attractive because it allows for the coherent excitation of only one or at most a few low-energy degrees of freedom, making control more precise. This stands in contrast to what can be achieved at near-visible wavelengths, where the excitation is highly incoherent, poorly selective, and induces heating.

A qualitative explanation for mode-selective optical control has been proposed by Först et al. [12-14], starting from the concepts of ionic Raman scattering [15-17]. In this description, the excitation of an infrared mode results in the displacement of the crystal structure along the coordinate of a second, anharmonically coupled Raman mode. The coherent energy transfer from the infrared to the Raman mode was analyzed through a lowest-order perturbative expansion of the lattice potential. This mechanism was termed "nonlinear phononics" and identified as a new type of coherent coupling between light and crystal lattices, beyond the extensively studied case of impulsive stimulated Raman scattering [18-20].

In this Rapid Communication, we present a microscopic theory for nonlinear phononics. This theory is based on symmetry considerations, as well as first-principles calculations of the energy surface combined with classical equations of motion in order to predict the dynamical path taken by the crystal lattice. We use this approach to study the response of two materials: the parent compound of the magnetoresistive manganites $\mathrm{PrMnO}_{3}$ (PMO), in relation to the experiments of Rini et al. [6], and that of the cuprate superconductors $\mathrm{La}_{2} \mathrm{CuO}_{4}$ (LCO).

At low fluence of the infrared excitation we show that, when considering nondegenerate phonon modes, it is only possible to displace the crystal lattice along a fully symmetric $A_{g}$ Raman mode. This makes use of an anharmonic cubic coupling of the form $Q_{\mathrm{IR}}^{2} Q_{\mathrm{R}}$, as envisioned by Först et al. [12-14]. We find that this applies to PMO, and by performing electronic structure calculations based on dynamical mean-field theory (DMFT), we provide a microscopic explanation for the lightinduced insulator-metal transition observed experimentally [6] in this material.

At larger fields, we predict a class of nonperturbative dynamics that involves Raman distortions of symmetry other than $A_{g}$. This dynamics, which becomes observable in LCO, originates from a quartic coupling of the type $Q_{\mathrm{IR}}^{2} Q_{\mathrm{R}}^{2}$. For these modes, a cubic coupling is forbidden by symmetry. Although at low fluence such a quartic coupling implies only a renormalization of the frequency of the Raman mode, an instability is found beyond a critical threshold, resulting in a distortion into a crystal structure of lower symmetry. Furthermore, we find that the near-threshold regime exhibits a dynamical stabilization of the crystal lattice, analogous to the Kapitza phenomenon in driven nonlinear systems [21,22].

We first consider PMO, which is an insulator with orthorhombic Pmna structure and four formula units per unit cell. In order to identify the nature and strength of the coupling between various IR and Raman phonons, we performed energy surface calculations as a function of the amplitude of these modes. The calculations were performed using density-functional theory in a plane-wave basis set (VASP code [23]). The 60 zone-center normal modes (see Supplemental Material for details [24]) were identified using 
the frozen-phonon method as implemented in the PHONOPY software package [25]. In the experiment of Rini et al., the IR mode corresponding to the stretching of the apical Mn-O bond was excited, but the frequency and symmetry of the excited mode was not analyzed. Hence, we used selection rules from group theory and explored several possible pairs of IR and Raman modes to infer which one might be relevant to the physics. We considered the excitation of the $B_{1 u}(54), B_{1 u}(56)$, $B_{2 u}(58)$, and $B_{3 u}(60)$ IR modes, the first one corresponding to the stretching of apical $\mathrm{Mn}-\mathrm{O}$ bonds and the others to in-plane ones. In the point group $\mathrm{mmm}$, the square of every irreducible representation is the $A_{g}$ representation. Hence, any of the seven $A_{g}$ modes of PMO can, in principle, have a nonlinear cubic coupling to the $B_{u}$ modes. We considered the coupling of all seven modes to the four aforementioned IR modes and found that the coupling between the apical Mn-O stretching mode $B_{1 u}(54)$ and the $A_{g}(9)$ Raman mode is substantial. Furthermore, as illustrated in Fig. 1 (top panel), a positive amplitude displacement of this Raman mode reduces the rotation of the $\mathrm{MnO}_{6}$ octahedra in the $a b$ plane. We show below that this favors the metallic state. Hence, we propose that the displacement of this mode through the nonlinear coupling to the pumped $B_{1 u}(54)$ mode is responsible for the effect observed by Rini et al. [6].

The calculated energy surface is displayed in Fig. 1, and fits the following expression, which involves a cubic anharmonic coupling between the two modes:

$$
\begin{aligned}
V\left(Q_{\mathrm{R}}, Q_{\mathrm{IR}}\right)= & \frac{1}{2} \Omega_{\mathrm{R}}^{2} Q_{\mathrm{R}}^{2}+\frac{1}{2} \Omega_{\mathrm{IR}}^{2} Q_{\mathrm{IR}}^{2}+\frac{1}{3} a_{3} Q_{\mathrm{R}}^{3} \\
& +\frac{1}{4} b_{4} Q_{\mathrm{IR}}^{4}-\frac{1}{2} g Q_{\mathrm{R}} Q_{\mathrm{IR}}^{2} .
\end{aligned}
$$

Here, $Q_{\mathrm{R}}$ and $Q_{\mathrm{IR}}$ are the displacements of the $A_{g}(9)$ and $B_{1 u}(54)$ modes, respectively. The calculated frequencies are $\Omega_{\mathrm{R}}=155 \mathrm{~cm}^{-1}$ and $\Omega_{\mathrm{IR}}=622 \mathrm{~cm}^{-1}$. The values of all coefficients are given in a table in the Supplemental Material [24]. From Fig. 1 (middle panel), one sees that, for a given value of the $B_{1 u}(54)$ amplitude, the energy landscape has a unique minimum as a function of the amplitude of the $A_{g}(9)$ mode. Furthermore, this mode is displaced from its equilibrium position as soon as the amplitude of the $B_{1 u}(54)$ mode is nonzero. This displacement being positive, it brings the structure closer to cubic symmetry (Fig. 1, top).

In order to substantiate that this can be responsible for the observed metallization of PMO, we have calculated the spectrum of electronic excitations of this compound in both the orthorhombic Pmna equilibrium structure and in the hypothetical cubic structure. This allows for a clear-cut comparison and for assessing the potential effect of fully undoing the orthorhombic distortion. The calculations are performed using the state-of-the art combination of electronic structure and dynamical mean-field theory (LDA + DMFT) [26-29] in order to properly account for the interplay between structural aspects and strong electronic correlations. As displayed in Fig. 1 (bottom panel), we find that PMO in the Pmna equilibrium structure is an insulator, in agreement with experiments, while the hypothetical cubic structure is, remarkably, a metal. As detailed in [24], the reason for this is the considerable reduction of the bandwidth of $\mathrm{Mn}-e_{g}$ states by the orthorhombic distortion, as compared to the cubic case in which the Mn-O-Mn bonds are straight, leading to a much
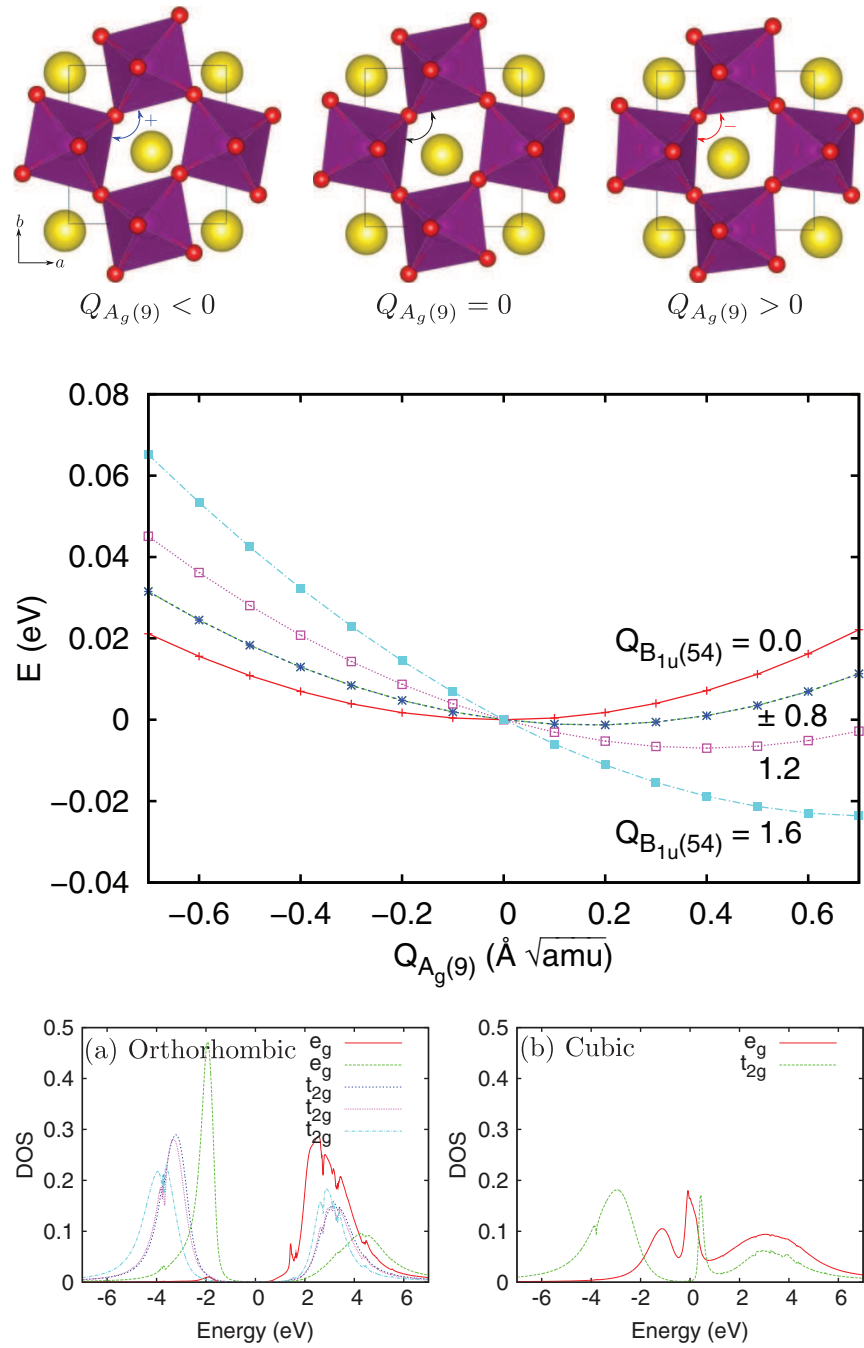

FIG. 1. (Color online) Top: Sketch of the atomic displacements corresponding to the $A_{g}(9)$ Raman mode in $\mathrm{PrMnO}_{3}$. A positive displacement drives the angle between octahedra (indicated by the arrow) closer to $90^{\circ}$, hence the structure closer to the cubic one. Middle: Total energy as a function of the $A_{g}(9)$ amplitude for several values of the $B_{1 u}(54)$ amplitude. For visual clarity, we plot $V\left(Q_{\mathrm{R}}, Q_{\mathrm{IR}}\right)-V\left(0, Q_{\mathrm{IR}}\right)$ so that all curves coincide at $Q_{\mathrm{R}}=0$. Bottom: LDA + DMFT orbitally resolved density of states (spectral function) of Mn- $d$ states in PMO for the equilibrium orthorhombic crystal structure (insulator) and cubic crystal structure (metal).

larger bandwidth. As a result, cubic PMO is found to be on the metallic side of the Mott transition, while orthorhombic PMO is found to be in the insulating side. This provides support to our proposal that the excitation of the $A_{g}$ Raman mode by pumping the $B_{1 u}$ IR modes explains the insulator-to-metal transition observed by Rini et al. [6].

We now turn to LCO, which forms in the orthorhombic $B m a b$ structure with two formula units per unit cell. We looked for coupling between high-frequency IR modes and low-frequency Raman modes, as relevant to low-frequency pump-probe experiments. We find that there is a substantial coupling between the $B_{1 g}(18)$ Raman and $B_{3 u}(41)$ IR modes of LCO. This IR mode also couples to a lower frequency $A_{g}(11)$ Raman mode, but the coupling is much smaller. An amplitude 

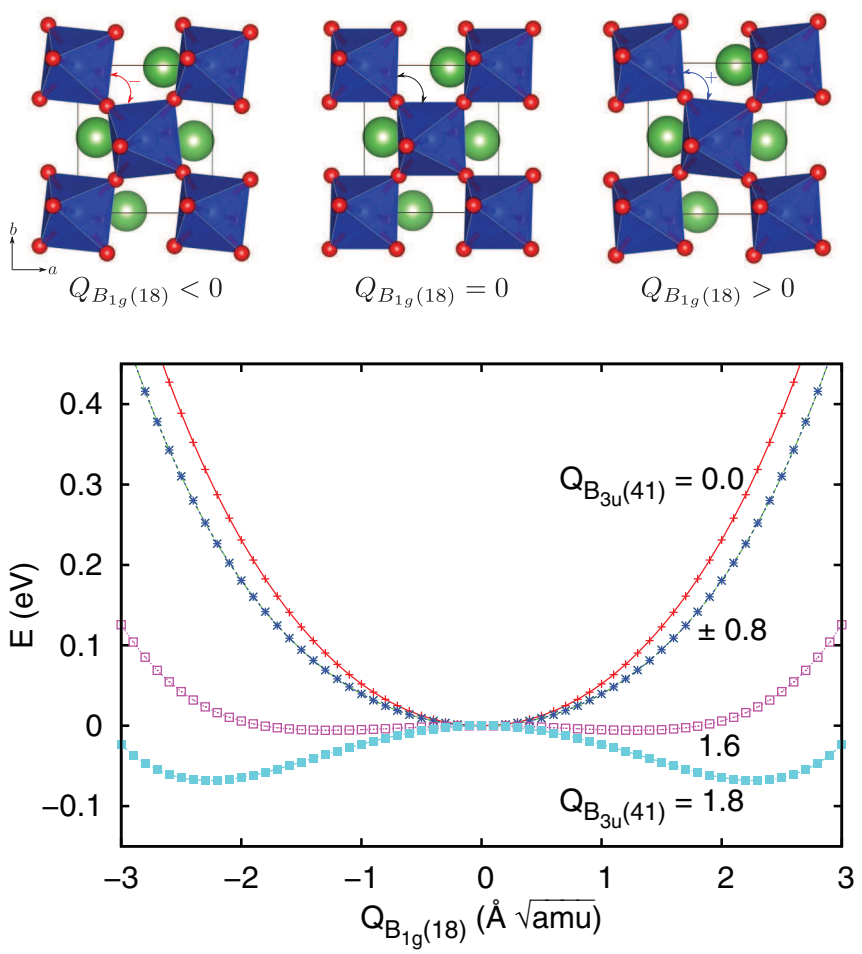

FIG. 2. (Color online) Top: Sketch of the atomic displacements corresponding to the $B_{1 g}(18)$ Raman mode in $\mathrm{La}_{2} \mathrm{CuO}_{4}$, illustrating the symmetry between a negative and positive displacement. Bottom: Total energy as a function of the $B_{1 g}(18)$ amplitude for several values of the $B_{3 u}(41)$ amplitude. For visual clarity, we plot $V\left(Q_{\mathrm{R}}, Q_{\mathrm{IR}}\right)-V\left(0, Q_{\mathrm{IR}}\right)$ so that all curves coincide at $Q_{\mathrm{R}}=0$.

of $2.0 \AA \sqrt{\mathrm{amu}}$ for the $B_{3 u}(41)$ mode shifts the minimum of the $A_{g}(11)$ mode to $-0.4 \AA \sqrt{\mathrm{amu}}$, whereas the same amplitude of the $B_{3 u}(41)$ mode generates minima at $\pm 2.4 \AA \sqrt{\mathrm{amu}}$ for the $B_{1 g}(18)$ mode. Hence, we only focus on the dynamics of the $B_{1 g}(18)$ and $B_{3 u}(41)$ modes. The $B_{1 g}(18)$ mode corresponds to the in-plane rotations of the $\mathrm{CuO}_{6}$ octahedra as shown in Fig. 2 (top) and the $B_{3 u}(41)$ involves in-plane stretching of the $\mathrm{Cu}-\mathrm{O}$ bonds. The $B_{1 g}(18)$ mode breaks the twofold rotational symmetry along the $x$ and $y$ axes as well as the reflection symmetry with the mirrors on the $x z$ and $y z$ planes. Therefore, the structures generated by the positive and negative $B_{1 g}(18)$ amplitudes are related by these symmetries.

The calculated energy surface for LCO is also displayed in Fig. 2 and fits the following expression [with $Q_{\mathrm{R}}$ and $Q_{\text {IR }}$ the amplitudes of the $B_{1 g}(18)$ and $B_{3 u}(41)$ modes, respectively, and the calculated frequencies $\Omega_{\mathrm{R}}=162$ and $\Omega_{\mathrm{IR}}=633 \mathrm{~cm}^{-1} \mathrm{]}$ :

$$
\begin{aligned}
V\left(Q_{\mathrm{R}}, Q_{\mathrm{IR}}\right)= & \frac{1}{2} \Omega_{\mathrm{R}}^{2} Q_{\mathrm{R}}^{2}+\frac{1}{2} \Omega_{\mathrm{IR}}^{2} Q_{\mathrm{IR}}^{2}+\frac{1}{4} a_{4} Q_{\mathrm{R}}^{4} \\
& +\frac{1}{4} b_{4} Q_{\mathrm{IR}}^{4}-\frac{1}{2} g Q_{\mathrm{R}}^{2} Q_{\mathrm{IR}}^{2} .
\end{aligned}
$$

In contrast to $\mathrm{PMO}$, we find a single-potential well around the equilibrium value for the $B_{1 g}(18)$ mode at small $B_{3 u}(41)$ amplitude. Remarkably, a double well is generated beyond a critical value of the $B_{3 u}(41)$ amplitude. In accord with the observation above, the energy is symmetric upon reversal of the sign of the $B_{1 g}(18)$ amplitude. The nonlinear coupling is here of the form $Q_{\mathrm{R}}^{2} Q_{\mathrm{IR}}^{2}$, consistent with this symmetry. We note that only the coupling to pairs of identical, zone-center Raman modes have been considered in the analysis of the $Q_{\mathrm{R}}^{2} Q_{\mathrm{IR}}^{2}$ coupling term. Coupling to pairs of Raman modes with opposite momenta away from zone center is, in principle, possible and should be considered in future work.

The disparate nature of the potential energy surface in PMO and LCO can be explained by symmetry considerations. Being associated with the trivial representation of the crystal symmetry group, an $A_{g}$ Raman mode does not break any symmetry. Hence, the atomic displacements associated with vibrations of this mode whose amplitude have equal magnitude but opposite sign are not related by symmetry. Their energies are in general different, resulting in terms with odd powers of $Q_{A_{g}}$. In contrast, the $B_{1 g}(18)$ mode breaks the rotational and mirror symmetries mentioned above. The structure that results from vibrations of the $B_{1 g}(18)$ mode with equal magnitude but opposite signs are related by symmetry. They thus have the same energy and only even powers of $Q_{B_{1 g}(18)}$ are allowed. Incidentally, only even powers of the IR mode are allowed in the total energy for similar symmetry reasons. For nondegenerate phonon modes, it can also be checked that the above form of the coupling between Raman and IR modes is consistent with the requirement that the product of the irreducible representations associated with all phonon modes involved in the coupling should contain the trivial $A_{g}$ representation. Indeed, $A_{g} \subset A_{g} \otimes B_{1 u} \otimes B_{1 u}$ for PMO, and $A_{g} \subset B_{1 g} \otimes B_{1 g} \otimes B_{3 u} \otimes B_{3 u}$ for LCO.

We finally discuss the dynamics of the nonlinearly coupled modes when the IR mode is pumped externally. We simplify the problem by treating the Raman and IR modes as two coupled classical oscillators. These oscillators are subject to a force deriving from the calculated energy surface [Eqs. (1) and (2)] and to a driving term $F(t)=F \sin (\Omega t) e^{-t^{2} / 2 \sigma^{2}}$, where $F$, $\sigma$, and $\Omega$ are the amplitude, width, and frequency of the light pulse, respectively. In the case of PMO (cubic coupling), the resulting equations of motion read

$$
\begin{aligned}
\ddot{Q}_{\mathrm{IR}}+\Omega_{\mathrm{IR}}^{2} Q_{\mathrm{IR}} & =g Q_{\mathrm{R}} Q_{\mathrm{IR}}-b_{4} Q_{\mathrm{IR}}^{3}+F(t), \\
\ddot{Q}_{\mathrm{R}}+\Omega_{\mathrm{R}}^{2} Q_{\mathrm{R}} & =\frac{1}{2} g Q_{\mathrm{IR}}^{2}-a_{3} Q_{\mathrm{R}}^{2} .
\end{aligned}
$$

Following [12], the resulting dynamical behavior can be easily understood in the impulsive limit $\sigma \ll 1 / \Omega$ (see the analysis in the Supplemental Material [24], which differs in details from that of [12]). On resonance, the IR mode undergoes a forced oscillation of amplitude $\propto F \Omega_{\mathrm{IR}} \sigma^{3}$. The effective forcing field for the Raman mode is $g Q_{\mathrm{IR}}^{2} / 2 \propto g F^{2} \Omega_{\mathrm{IR}}^{2} \sigma^{6}\left(1-\cos 2 \Omega_{\mathrm{IR}} t\right)$ which has a rectified nonzero average value. Hence, the Raman mode oscillates around a displaced position as a result of the excitation of the IR mode by the light pulse, consistent with the displaced minimum of the energy surface (Fig. 1). The displacement occurs for an arbitrarily small pump amplitude, grows quadratically as $\propto g Q_{\mathrm{IR}, \max }^{2} / \Omega_{\mathrm{R}}^{2} \propto g F^{2} \sigma^{6} \Omega_{\mathrm{IR}}^{2} / \Omega_{\mathrm{R}}^{2}$, and the oscillation frequency $\left(\Omega_{R}\right)$ is unchanged by the light pulse. This behavior is also confirmed by numerical integration of these equations for a finite pulse width [24].

In the case of LCO (quartic coupling), the equations of motion read

$$
\begin{aligned}
\ddot{Q}_{\mathrm{IR}}+\Omega_{\mathrm{IR}}^{2} Q_{\mathrm{IR}} & =g Q_{\mathrm{R}}^{2} Q_{\mathrm{IR}}-b_{4} Q_{\mathrm{IR}}^{3}+F(t), \\
\ddot{Q}_{\mathrm{R}}+\Omega_{\mathrm{R}}^{2} Q_{\mathrm{R}} & =g Q_{\mathrm{R}} Q_{\mathrm{IR}}^{2}-a_{4} Q_{\mathrm{R}}^{3} .
\end{aligned}
$$



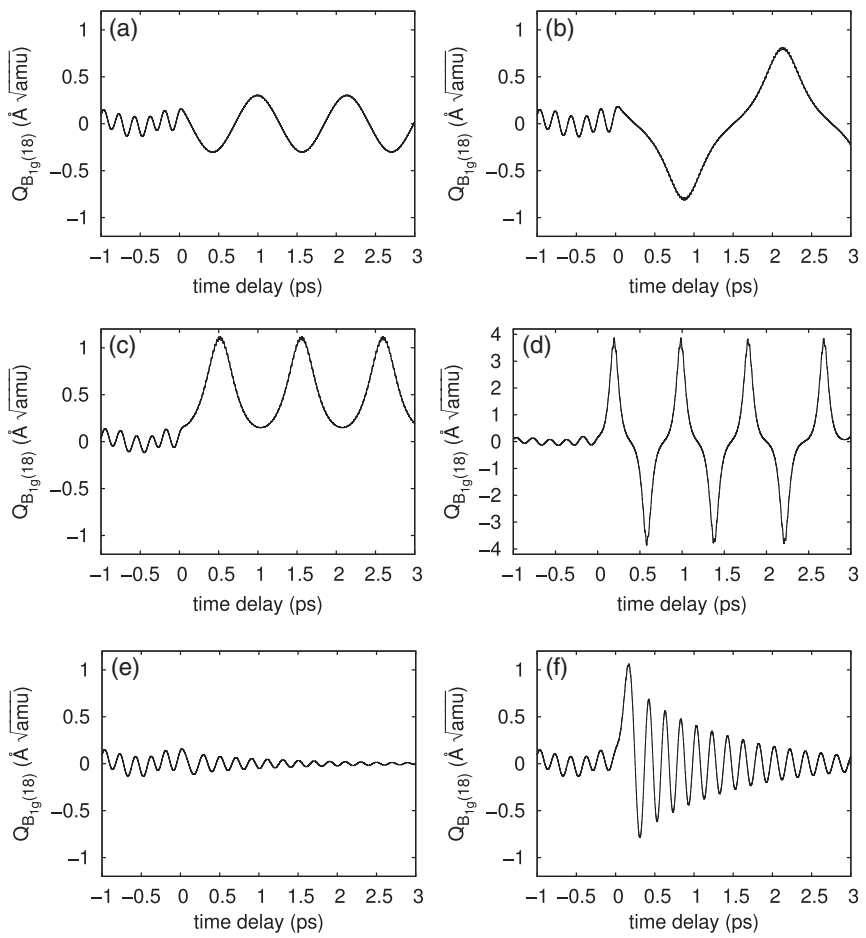

FIG. 3. Dynamics of the Raman mode in the case of a quartic coupling (as in LCO). Top panel: The four dynamical regimes in the undamped case [(a)-(d)]. Bottom panel: Dynamics below (e) and above threshold (f) in the presence of damping.

Numerical integration of these equations reveal a much richer behavior than in the cubic case, as illustrated in Fig. 3. There is a threshold value $F_{c}$ of the pulse amplitude below which the Raman mode is not displaced and oscillates around its original equilibrium position. In this regime [Fig. 3(a)], the period of oscillation is amplified by the light pulse, as well as its amplitude. Upon increasing $F>F_{c}$ above threshold, three different regimes are successively found [Figs. 3(b), 3(c), and 3(d)]. In a narrow range of $F \gtrsim F_{c}$, a long-period oscillation reaching out to the two wells of the double-well potential is found (b). This is also the case (d) at large values of $F$ but with a much shorter period. For an intermediate range of values of $F>F_{c}$, a rectified regime (c) is again found, in which the Raman mode oscillates around a displaced position.

Here also, some analytical understanding can be achieved in the impulsive limit, as detailed in [24]. It is immediately apparent from the second equation in (4) that the IR mode does not act as a forcing term in this case, but rather as a time-dependent modulation of the frequency of the Raman mode $\Omega_{\mathrm{R}}^{2} \rightarrow \Omega_{\mathrm{R}}^{2}\left[1-g Q_{\mathrm{IR}}^{2}(t) / \Omega_{\mathrm{R}}^{2}\right]$. Hence, for $F<F_{c}$, the dynamics is well approximated by a Mathieu equation describing a parametric oscillator. The threshold amplitude $F_{c}$ can be analytically calculated in the impulsive limit from known properties of this equation and is given by the condition $g Q_{\mathrm{IR}, \max }^{2} / \Omega_{\mathrm{R}}^{2}=2$, where $Q_{\mathrm{IR}, \max } \propto F \Omega_{\mathrm{IR}} \sigma^{3}$ is the amplitude of the excited IR mode. Remarkably, $F_{c}$ is $\sqrt{2}$ times larger than the value (corresponding to $g Q_{\mathrm{IR}, \max }^{2} / \Omega_{\mathrm{R}}^{2}=1$ ) at which the energy landscape (2) develops a double well and the original equilibrium position becomes unstable from a static viewpoint. Hence, in the range $F_{c} / \sqrt{2}<F<F_{c}$, there is a dynamical stabilization of the unrectified oscillatory motion, analogous to the Kapitza phenomenon for a vibrating pendulum [21,22]. The oscillation frequency in this regime is reduced by the light pulse according to $\Omega_{\text {eff }}^{2}=\Omega_{\mathrm{R}}^{2}\left[1-\left(F / F_{c}\right)^{2}\right]$, in accordance with the numerical observations.

The critical amplitude of the $B_{3 u}(41)$ mode associated with the dynamical threshold for LCO is found to be $Q_{\mathrm{IR}, \max }^{c} \simeq 1.6 \AA \sqrt{\mathrm{amu}}$. This corresponds to a typical in-plane displacement of the oxygen atoms of $0.276 \AA$. Previous experiments [7] have shown that atomic displacements of that magnitude can be induced by available light pulses, so that this effect should indeed be observable. We note furthermore [24] that the in-plane rotations associated with the $B_{1 g}(18)$ Raman mode lead to a reduction of the bandwidth of the $\mathrm{Cu}$ $d_{x^{2}-y^{2}} / \mathrm{O}-p_{x, y}$ antibonding band. This could be used to modify the correlation strength and superexchange coupling in this cuprate.

We have also considered the effect of damping on this dynamical behavior, as displayed in Figs. 3(e) and 3(f). For typical values (5\%-10\% of the linewidth) of the damping of the IR mode, the Raman mode is found to relax back to an oscillating behavior around its original equilibrium position, as expected. However, the salient features of the above dynamical regimes are preserved. In particular, a critical value of the pump amplitude is still present, above which the pump excites a coherent oscillation of the Raman mode, with an initial largeamplitude displacement [Fig. 3(f)].

In summary we have developed a microscopic theory of the light control of crystal lattices by strong field $\mathrm{THz}$ radiation. Our theory greatly expands the understanding of this class of phenomena, explaining many of the observations reported to date. The cubic anharmonic coupling between IR and Raman modes, already used to explain experimental results qualitatively, is shown here to apply only to totally symmetric modes (in the nondegenerate case considered here). For manganites, we combine first-principles structural studies and electronic structure calculations, and identify the microscopic pathway for the insulator-to-metal transition observed experimentally. More importantly, we predict a regime for which the light can be used to initiate dynamical symmetry breaking. This latter class of phenomena is nonperturbative, involves a quartic coupling, and leads to transient mode softening and dynamic stabilization. We show that this second regime is dominant in specific crystal structures, and predict that its experimental observation is possible with current technology.

We are thankful to Leonid Pourovskii and Michel Ferrero for assistance with the LDA + DMFT calculations, and to Roman Mankowski, Michael Först, Roberto Merlin, and JeanMarc Triscone for discussions and suggestions. This work was supported by a grant (Grant No. ERC-319286 QMAC) from the European Research Council and by the Swiss National Supercomputing Centre (CSCS) under project ID s404. 
[1] K. Miyano, T. Tanaka, Y. Tomioka, and Y. Tokura, Phys. Rev. Lett. 78, 4257 (1997).

[2] A. Cavalleri, C. Tóth, C. W. Siders, J. A. Squier, F. Ráksi, P. Forget, and J. C. Kieffer, Phys. Rev. Lett. 87, 237401 (2001).

[3] S. Iwai, S. Tanaka, K. Fujinuma, H. Kishida, H. Okamoto, and Y. Tokura, Phys. Rev. Lett. 88, 057402 (2002).

[4] M. Chollet, L. Guerin, N. Uchida, S. Fukaya, H. Shimoda, T. Ishikawa, K. Matsuda, T. Hasegawa, A. Ota, H. Yamochi et al., Science 307, 86 (2005).

[5] L. Perfetti, P. A. Loukakos, M. Lisowski, U. Bovensiepen, H. Berger, S. Biermann, P. S. Cornaglia, A. Georges, and M. Wolf, Phys. Rev. Lett. 97, 067402 (2006).

[6] M. Rini, R. Tobey, N. Dean, J. Itatani, Y. Tomioka, Y. Tokura, R. W. Schoenlein, and A. Cavalleri, Nature (London) 449, 72 (2007).

[7] R. I. Tobey, D. Prabhakaran, A. T. Boothroyd, and A. Cavalleri, Phys. Rev. Lett. 101, 197404 (2008).

[8] A. D. Caviglia, R. Scherwitzl, P. Popovich, W. Hu, H. Bromberger, R. Singla, M. Mitrano, M. C. Hoffmann, S. Kaiser, P. Zubko et al., Phys. Rev. Lett. 108, 136801 (2012).

[9] D. Fausti, R. Tobey, N. Dean, S. Kaiser, A. Dienst, M. Hoffmann, S. Pyon, T. Takayama, H. Takagi, and A. Cavalleri, Science 331, 189 (2011).

[10] W. Hu, S. Kaiser, D. Nicoletti, C. R. Hunt, I. Gierz, M. C. Hoffmann, M. Le Tacon, T. Loew, B. Keimer, and A. Cavalleri, Nat. Mater., doi:10.1038/nmat3963 (2014).

[11] S. Kaiser, C. R. Hunt, D. Nicoletti, W. Hu, I. Gierz, H. Y. Liu, M. Le Tacon, T. Loew, D. Haug, B. Keimer, and A. Cavalleri, Phys. Rev. B 89, 184516 (2014).

[12] M. Först, C. Manzoni, S. Kaiser, Y. Tomioka, Y. Tokura, R. Merlin, and A. Cavalleri, Nat. Phys. 7, 854 (2011).

[13] M. Först, R. I. Tobey, S. Wall, H. Bromberger, V. Khanna, A. L. Cavalieri, Y.-D. Chuang, W. S. Lee, R. Moore, W. F. Schlotter et al., Phys. Rev. B 84, 241104(R) (2011).
[14] M. Först, R. Mankowsky, H. Bromberger, D. M. Fritz, H. Lemke, D. Zhu, M. Chollet, Y. Tomioka, Y. Tokura, R. Merlin et al., Solid State Commun. 169, 24 (2013).

[15] A. A. Maradudin and R. F. Wallis, Phys. Rev. B 2, 4294 (1970).

[16] R. F. Wallis and A. A. Maradudin, Phys. Rev. B 3, 2063 (1971).

[17] T. P. Martin and L. Genzel, Phys. Status Solidi B 61, 493 (1974).

[18] L. Dhar, J. A. Rogers, and K. A. Nelson, Chem. Rev. 94, 157 (1994).

[19] R. Merlin, Solid State Commun. 102, 207 (1997).

[20] T. Dekorsy, G. Cho, and H. Kurz, Light Scattering in Solids VIII (Springer, Berlin, 2000), Vol. 76.

[21] P. L. Kapitza, Sov. Phys. JETP 21, 588 (1951).

[22] A. Stephenson, Philos. Mag. 15, 233 (1908).

[23] G. Kresse and J. Furthmüller, Phys. Rev. B 54, 11169 (1996).

[24] See Supplemental Material at http://link.aps.org/supplemental/ 10.1103/PhysRevB.89.220301 for detailed methods, phonon frequencies, nonlinear couplings, analytical study of the dynamical behavior, and additional results of the electronic structure calculations.

[25] A. Togo, F. Oba, and I. Tanaka, Phys. Rev. B 78, 134106 (2008).

[26] A. Georges, G. Kotliar, W. Krauth, and M. J. Rozenberg, Rev. Mod. Phys. 68, 13 (1996).

[27] G. Kotliar, S. Y. Savrasov, K. Haule, V. S. Oudovenko, O. Parcollet, and C. A. Marianetti, Rev. Mod. Phys. 78, 865 (2006).

[28] M. Aichhorn, L. Pourovskii, V. Vildosola, M. Ferrero, O. Parcollet, T. Miyake, A. Georges, and S. Biermann, Phys. Rev. B 80, 085101 (2009).

[29] M. Ferrero and O. Parcollet, TRIQS: A Toolbox for Research in Interacting Quantum Systems, http://ipht.cea.fr/triqs 